\title{
Mesenteritis esclerosante. A propósito de dos casos con diferente forma de presentación clínica
}

\author{
P. MARTÍNEZ ODRIOZOLA, N. GARCÍA JIMÉNEZ, S. CABEZA GARCÍA, \\ E. OCEJA BARRUTIETA ${ }^{1}$
}

Servicios de Medicina Interna y ${ }^{\prime}$ Hospitalización a Domicilio.

Hospital de Basurto. Bilbao

SCLEROSING MESENTERITIS. REPORT OF TWO CASES WITH DIFFERENT CLINICAL PRESENTATION

\section{RESUMEN}

La mesenteritis esclerosante es una rara enfermedad del mesenterio, de causa desconocida y de evolución crónica, que tiene dos formas de presentación: paniculitis mesentérica y mesenteritis retráctil; en la primera predomina la inflamación de la grasa mesentérica y en la segunda el engrosamiento fibrótico con acortamiento del mesenterio. Presentamos dos casos de mesenteritis esclerosante cuya forma de presentación clínica fue muy diferente. Uno de los pacientes debutó con un cuadro de suboclusión intestinal y el segundo caso ingresó para estudio de una fiebre de origen desconocido (FOD). Mientras que la obstrucción intestinal es una complicación relativamente frecuente, la presentación de esta enfermedad como FOD es excepcional.

PALABRAS CLAVE: Mesenteritis esclerosante. Paniculitis mesentérica. Fiebre de origen desconocido.

\begin{abstract}
Sclerosing mesenteritis is a rare disease of the mesenterium of unknown cause and chronic evolution. Two presentations have been reported: mesenteric panniculitis and retractile mesenteritis; inflammation of mesenteric fat predominates in one of them, while fibrotic thickening and shortening of the mesenterium is characteristic of the other. We report two cases of sclerosing mesenteritis which presented in very different ways. One patient suffered an episode of intestinal obstruction, and the second one was admitted for evaluation of fever of unknown origin $(F U O)$. While intestinal obstruction is a relatively common complication of sclerosing mesenteritis, its presentation as a FUO is exceptional.
\end{abstract}

KEY WORDS: Sclerosing mesenteritis. Mesenteric panniculitis. Fever of unknown origin.

Martínez Odriozola P, García Jiménez N, Cabeza García S, Oceja Barrutieta E. Mesenteritis esclerosante. A propósito de dos casos con diferente forma de presentación clínica. An Med Interna (Madrid) 2003; 20: 254-256.

\section{INTRODUCCIÓN}

La mesenteritis esclerosante es una enfermedad de causa desconocida y presentación poco frecuente, caracterizada por una extensa inflamación crónica inespecífica del mesenterio del intestino delgado. Es una enfermedad poco conocida y que se ha denominado de múltiples formas (paniculitis mesentérica, lipodistrofia mesentérica, lipogranulomatosis esclerosante, mesenteritis retráctil, fibromatosis mesentérica, lipodistrofia intestinal, lipogranuloma del mesenterio), lo que ha aumentado aún más la confusión del cuadro. El diagnóstico es siempre histológico y en la actualidad se distinguen dos formas anatomo-clínicas: la paniculitis mesentérica y la mesenteritis retráctil; en la primera predomina la inflamación de la grasa mesentérica y en la segunda el engrosamiento fibrótico con acortamiento del mesenterio, provocando con frecuencia una obstrucción intestinal (1).
En el presente artículo describimos dos casos de mesenteritis esclerosante cuya forma de presentación clínica fue muy diferente. Uno de los pacientes debutó con un cuadro de suboclusión intestinal y el segundo caso ingresó para estudio de una fiebre de origen desconocido (FOD).

\section{CASOS APORTADOS}

Caso 1. Paciente de 42 años con antecedentes de exposición laboral a vinilos y apendicectomía, que seis meses antes de su ingreso en nuestra unidad precisó asistencia hospitalaria por dolor abdominal con diagnóstico al alta de crisis suboclusiva. Ingresó por dolor periumbilical de dos meses de evolución, con episodios de febrícula intermitente, de predominio vespertino, acompañada de sudoración y adelgazamiento de nueve $\mathrm{kg}$, sin anorexia ni astenia. La exploración física se encontraba dentro de los límites de la normalidad. Entre los

Trabajo aceptado: 23 de mayo de 2002 
datos de laboratorio destacaban VSG de $80 \mathrm{~mm}$, proteína C reactiva de $12,8 \mathrm{mg} / \mathrm{dl}$ (VR 0-0,5), alfa 2-globulina $0,9 \mathrm{~g} / \mathrm{dl}$, fibrinógeno 885 $\mathrm{mg} / \mathrm{dl}$. Fueron normales o negativas las siguientes determinaciones: hemograma, glucosa, urea, creatinina, aminotransferasas, F. Alcalina, gammaGT, bilirrubina, LDH, Na, K, Ca, sangre en heces, CEA, CA 19.9, alfa-fetoproteína, beta 2-microglubulina, anticuerpos antinucleares, complemento, factor reumatoide, amilasas, tuberculina, urocultivo, hemocultivos, serología de brucella, salmonella, toxoplasma, lúes, VIH, VHB, VHC y VEB, y la investigación de parásitos, Ziehl y Löwenstein en heces. En las radiografías de tórax, abdomen, ecografía pélvica, tránsito intestinal, TC (tomografía computarizada) de tórax y gammagrafía con galio no se encontró anomalía relevante alguna. En la TC abdominal (Fig. 1) se apreciaron cuatro adenopatías de $15 \mathrm{~mm}$ de diámetro en localización retroperitoneal, paraaórtica, interaortocava y raíz de mesenterio, más afectación tipo masa mal definida afectando la grasa en torno al pedículo vascular central del mesenterio. En la endoscopia digestiva alta se detectó una hernia hiatal no complicada. Se realizó laparotomía exploradora, en la que se pudo observar imagen de masa que afectaba la grasa mesentérica proximal a un asa yeyunal. Los resultados de las biopsias fueron para las adenopatías de adenitis granulomatosa no necrotizante, con Ziehl y Löwenstein negativos, y para la grasa mesentérica de tejido fibroadiposo con infiltración granulomatosa no necrotizante, realizándose el diagnóstico de mesenteritis esclerosante. El paciente fue tratado con antiinflamatorios no esteroideos obteniéndose respuesta parcial y el tratamiento con prednisona por vía oral, a dosis de $1 \mathrm{mg} / \mathrm{kg} /$ día, no consiguió resolver el proceso. Tras asociar colchicina el paciente mejoró clínica, analítica y radiológicamente. Tres años después del inicio de su proceso se mantiene estable y asintomático, continuando tratamiento con prednisona (5 $\mathrm{mg} /$ día) y colchicina ( $1 \mathrm{mg} /$ día $)$.

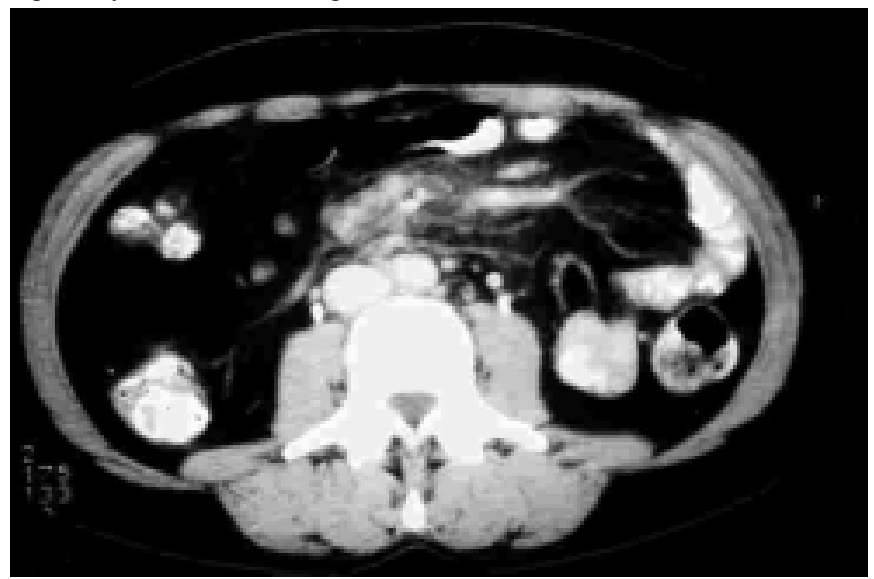

Fig. 1. Tomografía computarizada en la que se detectan adenopatías en localización retroperitoneal, paraaórtica, interaortocava y raíz de mesenterio y engrosamiento de la grasa en torno al pedículo vascular central del mesenterio.

Caso 2. Mujer de 59 años de edad, sin antecedentes de interés, que consultó por cuadro febril de tres semanas de evolución, con severa afectación del estado general y sin otra sintomatología acompañante. La exploración física no aportó datos patológicos. Entre los datos de laboratorio destacaban una anemia normocítica (Hb de 11,1 $\mathrm{g} / \mathrm{dl}$ ), VSG de $75 \mathrm{~mm}$ a la $1^{\text {a }}$ hora, GOT $59 \mathrm{U} / \mathrm{I}$, GPT $89 \mathrm{U} / \mathrm{I}$, GGT $140 \mathrm{U} / \mathrm{I}, \mathrm{F}$. Alcalina $654 \mathrm{U} / \mathrm{l}$, alfa 2-globulina $1,17 \mathrm{~g} / \mathrm{dl}$ y fibrinógeno $805 \mathrm{mg} / \mathrm{dl}$. Cultivos de sangre, orina y heces negativos. Micobacterias en sangre, esputo y orina negativos. Tuberculina PPD negativa. Serología de hepatitis, brucella, mononucleosis, citomegalovirus y VIH negativa. Anticuerpos antinucleares negativos. Hormonas tiroideas y beta 2-microglobulina normales. La radiografía de tórax, TC de tórax y ecografía abdominal fueron normales. La TC de abdomen demostró un engrosamiento difuso de la grasa en la raíz del mesenterio y la presencia de múltiples adenopatías de pequeño tamaño en retroperitoneo y raíz del mesenterio. Se realizó laparoscopia diagnóstica, en la que se observó un engrosamiento difuso de la raíz del mesenterio con adenopatías en su interior. En el examen histológico de las adenopatías se observaron cambios reactivos inespecíficos y el mesenterio mostraba un tejido adiposo tabicado por septos fibrosos y con infiltrado inflamatorio crónico, compatible con mesenteritis esclerosante. El tejido hepático no tenía alteraciones destacables. La paciente fue tratada con prednisona vía oral, a dosis de $1 \mathrm{mg} / \mathrm{kg} / \mathrm{d}$ á, siendo la evolución clínica favorable, con desaparición de la fiebre y normalización de los parámetros de laboratorio. El tratamiento fue suspendido seis meses después, sin que se observaran recaídas.

\section{DISCUSIÓN}

La mesenteritis esclerosante es una rara enfermedad del mesenterio, de causa desconocida y de evolución crónica, que aparece a partir de la quinta década de la vida y más frecuente en el sexo masculino. Actualmente se distinguen dos formas histológicas, paniculitis mesentérica y mesenteritis retráctil, aunque para algunos autores (1) esta última podría representar una forma fibrótica extrema de la primera. La paniculitis mesentérica se caracteriza por una infiltración del mesenterio por macrófagos espumosos cargados de lípidos, asociada a una fibrosis leve-moderada, lo que da lugar a un engrosamiento difuso del mesenterio o a la presencia de masas mesentéricas únicas o múltiples. Las manifestaciones clínicas más frecuentes son la masa abdominal palpable, el dolor abdominal, la fiebre y la pérdida de peso, aunque alrededor del $40 \%$ de los pacientes están asintomáticos (1-4). En la mesenteritis retráctil se observa un mesenterio engrosado con abundantes adherencias al peritoneo. El intestino delgado es retraído hacia la raíz del mesenterio, originándose estenosis que provocan dilatación de asas. La linfadenopatía regional es frecuente. El examen histológico muestra proliferación de tejido fibroso con bandas de colágena que reemplazan la grasa mesentérica normal. La manifestación clínica más frecuente es la obstrucción intestinal, parcial o completa (1). La mesenteritis esclerosante se asocia con frecuencia a otras enfermedades, especialmente el linfoma, la fibrosis retroperitoneal, el mesotelioma pleural, el síndrome de Gardner y la úlcera de recto (3).

Para el diagnóstico de esta enfermedad la técnica de imagen más utilizada es la TC abdominal (3), con la que se puede observar el engrosamiento o las masas del mesenterio, que aunque sugestivas son imágenes inespecíficas, por lo que el diagnóstico definitivo siempre se hará mediante examen histológico del mesenterio, con muestras obtenidas mediante laparoscopia o laparotomía exploradora. El diagnóstico diferencial de la paniculitis mesentérica se debe hacer con neoplasias malignas (carcinomatosis peritoneal, liposarcoma, linfoma), pseudotumor inflamatorio del peritoneo, necrosis grasa mesentérica de la pancreatitis aguda, enfermedad de Crohn y con la enfermedad de Weber-Christian (paniculitis generalizada); la mesenteritis retráctil se deberá diferenciar del linfoma, carcinoide, fibrosis retroperitoneal, enfermedad de Whipple y de la peritonitis fibrosante secundaria (traumatismos, cirugía, administración de beta-bloqueantes), además de las demás causas de obstrucción intestinal $(3,5)$. El pronóstico es incierto, aunque generalmente es una enfermedad de curso benigno. El tratamiento se recomienda únicamente en las formas sinto- 
máticas y se basa en la administración de glucocorticoides, solos o asociados a colchicina o inmunosupresores (azatioprina, ciclofosfamida) (6).

Los dos pacientes comentados en el presente artículo tuvieron una forma de presentación clínica muy diferente; mientras que el primer caso debutó con la clásica sintomatología de dolor abdominal y crisis de suboclusión intestinal, el segundo se presentó como fiebre de origen desconocido (FOD), no sufriendo dolor abdominal en ningún momento de su evolución. Aunque la fiebre en la mesenteritis esclerosante puede aparecer hasta en el $25 \%$ de los pacientes (1), es excepcional que sea su principal manifestación; de hecho, en la revisión de la literatura realizada por nosotros sólo hemos podido encontrar tres casos (7-9) que se presentaron clínica-

\section{Bibliografía}

1. Kipfer RE. Paniculitis mesentérica (lipodistrofia) y mesenteritis retráctil. En: Berk JE, Haubrich WS, Kalser MH, Roth JLA, Schaffner F, eds. Bockus, Gastroenterología (4 ${ }^{\mathrm{a}}$ ed). Barcelona: Salvat Editores SA, 1987: 4581-4585.

2. Phillips RH, Carr RA, Preston R, Pereira SP, Wilkinson ML, O’Donell PJ, et al. Sclerosing mesenteritis involving the pancreas: two cases of a rare cause of abdominal mass mimicking malignancy. Eur J Gastroenterol Hepatol 1999; 11: 1323-1329.

3. Sabaté JM, Torrubia S, Maideu J, Franquet T, Monill JM, Pérez C. Sclerosing mesenteritis. Imaging findings in 17 patients. AJR 1999; 172: 625-629.

4. Suriñach JM, Alegre J, Allende H, Alemán C, Jufresa J, Fernández de Sevilla T. Severa pérdida de peso en el debut de una paniculitis mesentérica. An Med Interna (Madrid) 1995; 12: 597-599. mente como FOD. Los datos de laboratorio eran inespecíficos, aunque ambos pacientes presentaron reactantes de fase aguda muy elevados. La biopsia mesénterica se realizó en un caso mediante laparotomía y en el otro mediante laparoscopia. Nosotros pensamos que, ante la sospecha de esta enfermedad, la técnica diagnóstica de elección es la laparoscopia, por tratarse de una cirugía mínimamente invasiva. En lo que respecta al tratamiento, también se observaron diferencias en ambos pacientes; en uno de ellos la respuesta terapéutica a la prednisona fue excelente, sin embargo, en el otro paciente fue necesaria la asociación de colchicina. En la actualidad, el tratamiento combinado de glucocorticoides y colchicina parece ser el indicado en las formas sintomáticas de la mesenteritis esclerosante (6), reservando los inmunosupresores para las

5. Kelly JK, Hwang WS. Idiopathic retractile (sclerosing) mesenteritis and its differential diagnosis. Am J Surg Pathol 1989; 13: 513-521.

6. Générau T, Bellin MF, Wechsler B, Huong LT, Bellanger J, Grellet J, et al. Demonstration of efficacy of combining corticosteroids and colchicine in two patients with idiopathic sclerosing mesenteritis. Dig Dis Sci 1996; 41: 648-688.

7. Sans M, Varas M, Anglada A, Bachs ME, Navarro S, Brugués J. Mesenteric panniculitis presenting as fever of unknown origin. Am J Gastroenterol 1995; 90: 1159-1161.

8. Arechaga S, Martín G, Alonso JM, San Román C, Serrano M. Paniculitis mesentérica. Rev Clin Esp 1983; 171: 347-350.

9. Otto F, Wedekind G. Mesenteric panniculitis. Z Gastroenterol 1991; 29: 395-397. 